\title{
Editorial for "Longitudinal cell-free DNA analysis in patients with small cell lung cancer reveals dynamic insights into treatment efficacy and disease relapse"
}

\author{
Michael Fleischhacker, Bernd Schmidt \\ Klinik für Innere Medizin, Schwerpunkt Pneumologie und Schlafmedizin, DRK Kliniken Berlin I Mitte, Berlin, Germany \\ Correspondence to: Michael Fleischhacker. Klinik für Innere Medizin, Schwerpunkt Pneumologie und Schlafmedizin, DRK Kliniken Berlin I Mitte, \\ Drontheimer Straße 39 - 40, 13359 Berlin, Germany. Email: fleischhackerm@drk-kliniken-berlin.de; michael.fleischhacker@uk-halle.de. \\ Comment on: Almodovar K, Iams WT, Meador CB, et al. Longitudinal Cell-Free DNA Analysis in Patients with Small Cell Lung Cancer Reveals \\ Dynamic Insights into Treatment Efficacy and Disease Relapse. J Thorac Oncol 2018;13:112-23.
}

Submitted Aug 17, 2018. Accepted for publication Sep 25, 2018.

doi: $10.21037 /$ tlcr.2018.09.19

View this article at: http://dx.doi.org/10.21037/tlcr.2018.09.19

Unfortunately, there are many reasons to assume that we will not see a marked decline in the number of people diagnosed with and dying from lung cancer any time soon. The reasons for that are manyfold. One very important factor is the strong association between smoking and development of lung cancer which holds especially true for small-cell lung cancer (SCLC). Due to an improved health education system, the number of smokers in some highincome countries like the US and others decreased over the last years. This is paralled by an unchanged or even increasing number of smokers in low to middle-income countries and in developing nations leading to a death toll of more than 6 million people annually. Additionally, there are other environmental factors like radon exposure, the handling of toxic chemicals, air pollution and to a lesser extent genetic factors which contribute to the development of lung cancer.

Recently there is growing interest in the application of liquid profiling methods (a term which might be better suited than "liquid biopsy") for the care of tumor patients in general and for lung cancer patients in particular. The last decade lead to the development of new and improved techniques for the sensitive and specific detection of tumorassociated genetic alterations in a high background of "healthy" DNA. Methods like real-time polymerase chain reaction (PCR), digital PCR, BEAMing, next-generation and long-read DNA sequencing made it possible to analyse not only traditionally used materials like genetic material from tumor tissues but from other sources like plasma, urine, different body liquids and lavage specimen as well. It was a long and arduous way from the first report which described the presence of extracellular nucleic acids in the plasma of humans (1) and the proof that it is possible to detect molecular genetic alterations associated with a tumor in the plasma of cancer patients $(2,3)$. This development culminated two years ago in the approval by the Food and Drug Administration (FDA) of the first blood test detecting gene mutations in the epidermal growth factor receptor $(E G F R)$ gene which is associated with non-small cell lung cancer (NSCLC) (4). This method is clinically relevant and used in different settings. When newly diagnosed patients demonstrate an activating EGFR mutation (no matter whether it was found in tissue or in plasma DNA) they are treated with a targeted therapy against these EGFR mutations instead of the standard platin-based chemotherapy. Very often these patients develop a resistance against the EGFR-inhibiting drugs based on additional mutations in this gene which prevent a binding of the drugs to the target. Frequently it is not possible to obtain tissue for a molecular genetic analysis and in this setting the extraction and examination of cell-free plasma DNA is a viable alternative. When such a resistance mutation, like the T790M mutation is found, the patients are treated with a third-generation tyrosine-kinase inhibitor (TKI) like osimertinib.

In contrast to NSCLC there are much less reports on the examination of extracellular nucleic acids in SCLC patients. From this point of view the paper by Almodovar 
and colleagues is to be welcomed (5). The authors used targeted next-generation sequencing to analyze cell-free plasma DNA from SCLC patients undergoing therapy. They looked for somatic variants in circulating cellfree DNA (cfDNA), i.e., single nucleotide variants, copy number alterations, and insertions or deletions in 14 genes that are frequently altered in SCLC, including TP53, RB1, BRAF, KIT, NOTCH1-4, PIK3CA, PTEN, FGFR1, MYC, $M Y C L 1$, and MYCN. Almodovar et al. demonstrated that the observed genetic alterations were closely associated with treatment response. Also, in some patients the analysis of cfDNA provided evidence of disease relapse before conventional imaging. As a proof of principle these are nice results but there are flies in the ointment.

First, $15 \%$ of the patients analysed are missed in the first place and could not be followed as no genetic alterations were found in their plasma DNA. This is surprising as one would expect that the selected panel of 14 genes detects almost all patients. Using a single marker only (methylation of the $S H O X 2$ gene) we were able to detect about the same percentage of lung cancer patients (NSCLC and SCLC) who underwent therapy. Based on these results we were able to divide the cohort of patients into groups of responders and non-responders with a very high probability already between 7 and 10 days after start of therapy (6). Therefore, it might be a good idea to modify the gene panel to be used in follow-up studies and to include other markers beside mutations like epigenetic alterations as well. This will hopefully enable the detection and characterization of all patients including the $15 \%$ missing in this analysis.

Second, according to the data supplied in the paper from none of the patients included in the study was a blood sample taken at the time of diagnosis, i.e., before start of therapy. It is possible that some of the markers which were used for the follow-up analysis disappeared during therapy leading to the $15 \%$ missing patients. We saw a similar effect in our pilot study in which all patients who were not treatment-naive before inclusion showed very low or zero mSHOX2 DNA plasma levels. This marker loss can be observed shortly after therapy start as was demonstrated by us as well as in other reports $(6,7)$.

Third, SCLC is characterized by a very aggressive course and a rather short life expectancy. Even patients diagnosed in a stage with a limited disease demonstrate a life expectancy which is not much better than for patients with an extended disease stage. Additionally, there are not many therapeutic choices in the first place and the options for patients who relapse (even after a successful initial therapy) are even smaller (in stark contrast to NSCLC patients). Therefore, the expectation that analyses like the one presented (even if performed in a prospective approach) might potentially be beneficial for SCLC patients is so far only a vague idea.

Fourth, as the authors mentioned there has not been much progress in the survival times for SCLC patients in the last decades and all efforts to prolong the survival so far failed (8). This is due in part to the large number of cells in S-phase fraction associated with a high metastatic potential. Whether targeted therapies including the newly developed immunotherapy approach will do a better job for SCLC patients than the standard chemotherapies in use so far has to be shown in the future.

Fifth, the time points at which blood was drawn do not follow a stringent guideline but are rather erratic. In order to define the best time points for blood drawing the schedule needs to be set ahead of time as part of the study protocol.

Sixth, SCLC patients (independent of their stage) show a very high relapse rate in the first 2 years after diagnosis. There are reports on SCLC patients who survived 5 or even 10 or more years and it would be very interesting to look for differences in their genetic makeup in these two populations (tissue could be a point to start with, if no plasma samples are available).

The points stated above let us to conclude that we are still far away from understanding the biology of SCLC which could be completely different than NSCLC tumors. And we need to know much more about the basic mechanisms of SCLC before we can answer the question whether the application of liquid profiling methods is beneficial for patients suffering from this disease.

\section{Acknowledgement}

None.

\section{Footnote}

Conflicts of Interest: The authors have no conflicts of interest to declare.

\section{References}

1. Mandel P, Metais P. Les acides nucléiques du plasma sanguin chez l'homme. C R Seances Soc Biol Fil 1948;142:241-3. 
2. Chen XQ, Stroun M, Magnenat JL, et al. Microsatellite alterations in plasma DNA of small cell lung cancer patients. Nat Med 1996;2:1033-5.

3. Nawroz H, Koch W, Anker P, et al. Microsatellite alterations in serum DNA of head and neck cancer patients. Nat Med 1996;2:1035-7.

4. The U.S. Food and Drug Administration. FDA approves first blood test to detect gene mutation associated with non-small cell lung cancer. Available online: https://www. fda.gov/newsevents/newsroom/pressannouncements/ ucm504488.htm

5. Almodovar K, Iams WT, Meador CB, et al. Longitudinal Cell-Free DNA Analysis in Patients with Small Cell Lung Cancer Reveals Dynamic Insights into Treatment Efficacy and Disease Relapse. J Thorac Oncol 2018;13:112-23.

6. Schmidt B, Beyer J, Dietrich D, et al. Quantification of cell-free mSHOX2 Plasma DNA for therapy monitoring in advanced stage non-small cell (NSCLC) and small-cell lung cancer (SCLC) patients. PLoS One 2015;10:e0118195.

7. Vallée A, Audigier-Valette C, Herbreteau G, et al. Rapid clearance of circulating tumor DNA during treatment with AZD9291 of a lung cancer patient presenting the resistance EGFR T790M mutation. Lung Cancer 2016;91:73-4.

8. Parikh M, Riess J, Lara PN Jr. New and emerging developments in extensive-stage small cell lung cancer therapeutics. Curr Opin Oncol 2016;28:97-103.
Cite this article as: Fleischhacker M, Schmidt B. Editorial for "Longitudinal cell-free DNA analysis in patients with small cell lung cancer reveals dynamic insights into treatment efficacy and disease relapse". Transl Lung Cancer Res 2018;7(Suppl 4):S367S369. doi: 10.21037/tlcr.2018.09.19 